\title{
Analysis of genetic and virulence variability of Stemphylium lycopersici associated with leaf spot of vegetable crops
}

\begin{abstract}
Stemphylium lycopersici (Enjoji) W. Yamam was initially described from tomato and has been reported to infect different hosts worldwide. Sequence analyses of the internal transcribed spacer (ITS) regions 1 and 2, including 5.8S rDNA (ITS-5.8S rDNA) and glyceraldehyde-3-phosphate dehydrogenase (gpd) gene, random amplified polymorphic DNA (RAPD) and inter-simple sequence repeat (ISSR), as well as virulence studies were conducted to analyze $46 \mathrm{~S}$. lycopersici isolates. Stemphylium lycopersici isolates used in this study were obtained from diseased tomato (Solanum lycopersicum L.), eggplant (Solanum melongena L.), pepper (Capsicum annuum L.) and lettuce (Lactuca sativa L.) from major vegetable growing regions of Malaysia, including the three states of Pahang, Johor and Selangor between 2011 and 2012. Phylogenetic analysis of a combined dataset of the ITS5.8S rDNA and gpd regions indicated that all isolates were clustered in the sub-cluster that comprised S. lycopersici, and were distinguished from other Stemphylium species. Cluster analyses using the UPGMA method for both RAPD and ISSR markers grouped S. lycopersici isolates into three main clusters with similarity index values of 67 and $68 \%$. The genetic diversity data confirmed that isolates of S. lycopersici are in concordance to host plants, and not geographical origin of the isolates. All S. lycopersici isolates were pathogenic on their original host plants and showed leaf spot symptoms; however, virulence variability was observed among the isolates. In cross-inoculation assays, the representative isolates were able to cause leaf spot symptoms on eggplant, pepper, lettuce and tomato, but not on cabbage.
\end{abstract}

Keyword: gpd; ISSR; ITS-5.8S rDNA; Phylogeny; RAPD 